\title{
Marble Track Music Sequencers for Children
}

\author{
Thomas Fischer \\ Wing Lau \\ School of Design
The Hong Kong Polytechnic University
Hung Hom, Kowloon, Hong Kong, China (SAR)
+852 27665478
sdtom@polyu.edu.hk
}

\begin{abstract}
We discuss the development of electronically enhanced construction kits for marble tracks that act as music sequencers for music education of young children. Marbles rolling along the tracks trigger sound events to produce the playback of musical notes and songs. Re-arrangeable tangible elements correspond to timing, durations and pitches of notes and thus allow electronic music sequencing by means of haptic programming. This approach to early music education separates the different tasks of melody making and instrument manipulation, aiming at faster success and fewer frustrating learning experiences. We present two marble track designs: an earlier one, which is based on a central synthesizer and a system-wide communication bus and a second one based on multiple decentralized synthesizer units. We discuss previous work in the field of machine-readable models and haptic programming and report on experiences with both sequencer designs including user tests of the second design.
\end{abstract}

\section{Keywords}

music education, sequencers, marble track, machinereadable models, haptic programming

\section{ACM Classification}

H.5.2 [User Interfaces]: Haptic I/O, H.5.5 [Sound and Music Computing]: Modeling

\section{BACKGROUND}

Learning to play music involves multiple simultaneous learning processes. At the very least, it requires a learner to apprehend the process of memorizing or reading and reproducing a given piece of music as well as the motor and co-ordination skills required to manipulate an instrument at an appropriate speed. Assuming that doing things separately rather than simultaneously is generally less difficult, we propose to deal with these different learning activities separately to hopefully allow easier and less frustrating early music learning experiences. With the exception of the most intuitive instruments such as the singing voice, the skills required for instrument manipulation pose a major stumbling block to the musical novice.
Programmable music sequencers potentially avoid this problem - but only for those who fathom their interfaces and interaction logic. In some step sequencer designs, musical events are programmed using spatial arrangements in two dimensions (figure 1). Nevertheless, sequencer technology available today is typically not accessible to young learners, for whom the understanding of abstract qualities and formal notations of music might be particularly challenging.

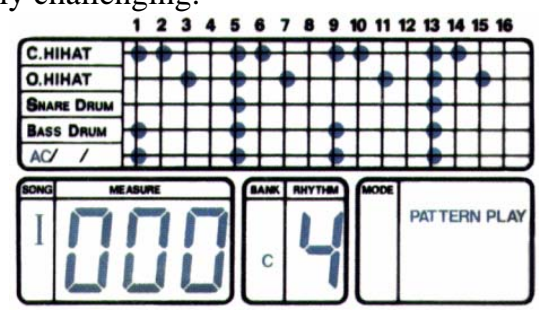

Fig. 1 Display of step sequencer Boss DR-100 by Roland

Our development of marble track music sequencers is based on a body of experience in developing machinereadable models [4], also known as tangible user interfaces [7]. Having originated from the field of computer-aided architectural design, and grounded in an understanding of architecture as logic states in space and time [3], machinereadable models provide ways to haptically configure and communicate spatially structured data as a means of input and output for digital computers [5]. The tangible user interfaces approach uses discrete physical tokens to represent digital data within physically confining constraints in order to provide intuitive user interfaces for given applications. Recent work in this field emphasizes the usefulness of such interfaces in child-computer interaction (see [6] pp. 382-384 and pp. 326-337). A limitation of existing toy-sized haptic music sequencers such as the Neurosmith Music Blocks is that they allow only simple variations of pre-programmed melody patterns. Physical input devices can be used to structure digital data not only in three but, depending on the mapping logic applied, also in more or fewer dimensions. One possible mapping of 3D arrangements to temporal procedures has previously been utilized in the development of an educational haptic programming environment for linedrawing robots [2] using machine-readable models. In this project, three-dimensional physical configurations were 
mapped onto ultimately sequential (hence one-dimensional) machine behavior. This aspect intuitively suggests the possibility of translating physical configurations of objects in space into sequential phenomena such as music playback.

This paper discusses how the three-dimensional (but logically, visually as well as acoustically clearly linear) haptic configuration of objects along a track allows for electronic music sequencing with an ease of use that is appropriate for school children, supporting their grasp of basic theoretical aspects of music such as note pitch and duration, melody making, time signatures and chords.

Some marble tracks that are currently commercially available contain short sequences of xylophone keys, which are however fixed and not reconfigurable. An earlier non-electronic marble-sequenced system for musical expression called the Musical Towers was designed in the early 1950s by the Eames Office in Santa Monica (Fig. 2). It consists of tall timber and Perspex structures with slots for interchangeable xylophone keys that are hit sequentially as one or more marbles drop through.

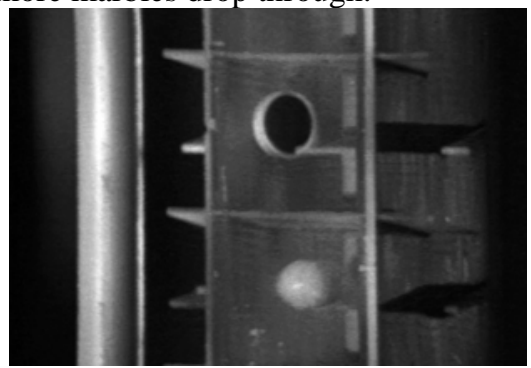

Fig. 2 Musical towers by Charles and Ray Eames [1]

Providing easy means to re-arrange notes on a time line and representing linear playback sequences in a very obvious way, the Musical Towers have some powerful properties for educational applications. But with several meters of height they are not necessarily the easiest and safest for children to operate and, in this respect, suggest the development of smaller and more accessible alternatives.

\section{INITIAL DESIGN}

An initial marble track implementation, shown in Figure 3 was designed and implemented as a student thesis project at our design school in spring of 2004. It consists of a base with four slots allowing the stacking of blocks to form four columns to vertically support inclined segments of the marble track. The track is made of two types of pipe segments. Clear PVC pipe segments, which passively determine the duration of individual notes, are not electronically enhanced but contain wires to connect power and data lines to the second type of pipe segments. These non-transparent, color-coded pipe segments are made of PVC pressure pipe connectors, each carrying a small microcontroller circuit. A passing marble triggers the circuits to play back notes through the base unit, which contains two stereo speakers, a controller circuit and a circuit board from a "hacked" toy music keyboard. The keyboard circuit is used for stereo sound synthesis and amplification, to provide a choice of 16 instrument sounds, volume control as well as a record-and-playback function. With one microcontroller and one pull-down resistor, the circuit in each note pipe is reduced to a minimum of two electronic components. The "marbles" used with this system are steel balls that were originally manufactured as ball bearings.

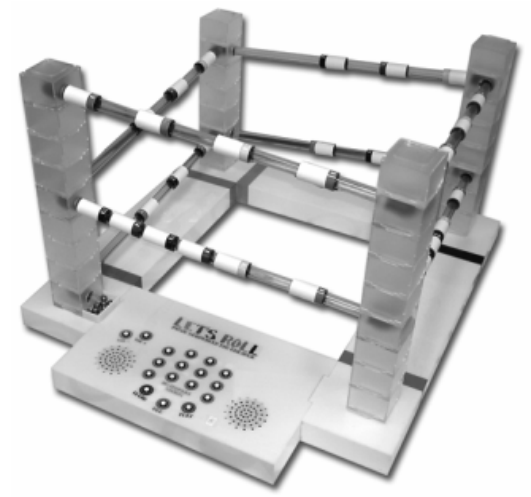

Fig. 3 First marble track design based on a system-wide communication bus and a centralized sound synthesizer.

The inside of each note pipe segment contains two bare wires between which a passing steel marble acts as a temporary switch, triggering the microcontroller to send a data message, encoding the respective note, to the base controller via a system-wide communication bus. We have specified a sparse serial protocol for this purpose which encodes 30 messages to encode a pitch range of $2 \frac{1}{2} 2$ octaves (30 notes). A microcontroller in the base interprets incoming signals and simulates the pressing of keys on the toy music keyboard circuit. This infrastructure allowed the reduction of the electrical connections along the marble track to three wires $(+5 \mathrm{~V}$, data and ground).

\section{REDESIGN}

Based on the experiences made with the first system we have designed and tested a second system. The key difference to the initial design is that it uses a decentralized set of attachable sound synthesizer modules, liberating it from the necessity for a system-wide communication bus. The marble track itself is one single object that does not require additional structural support units. Two parallel steel helices act as the actual track as well as the power supply lines to attachable synthesizer units. The helices are supported by a transparent Perspex frame, which can be read and utilized as 16 bar markers. Synthesizer units (roughly $7 \mathrm{~cm} \mathrm{x} 5 \mathrm{~cm} \times 3 \mathrm{~cm}$ in size) can carry up to four attachable color-coded physical tokens, which represent musical notes and percussion elements. Each synthesizer module is fitted with a melody processor capable of polyphonic sound synthesis. With up to four tokens attachable to each synthesizer unit, each unit supports 
polyphonic effects such as chords of three notes plus a percussion sound simultaneously.

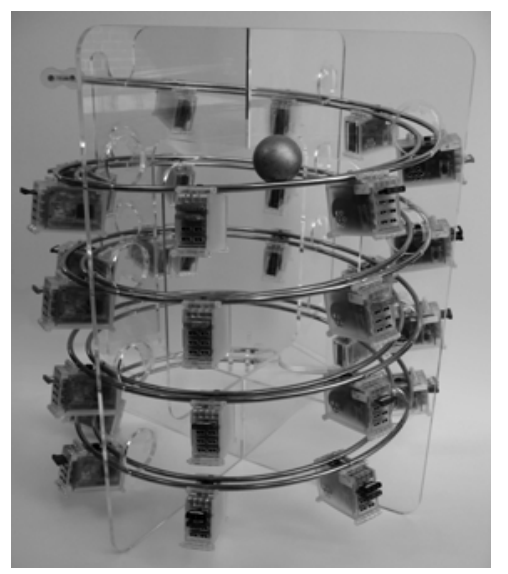

Fig. 4 Second design based on decentralized synthesizer modules with attachable note tokens

The decentralized synthesis architecture prohibits some functional aspects present in the initial design such as central sound and volume control as well as a recording function (the latter function seems dispensable since as a sequencer the system implicitly supports repeated playback by rolling a marble).

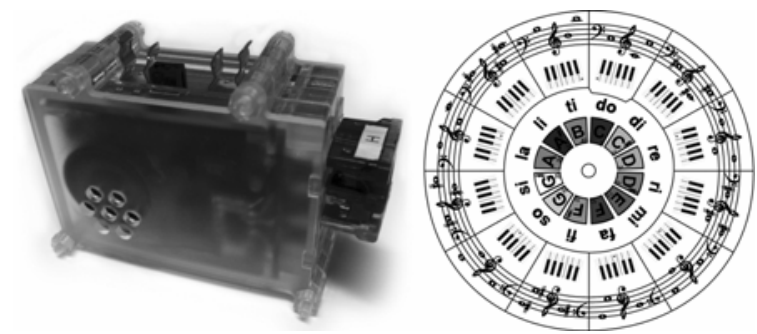

Fig. 5 Synthesizer unit with three note tokens attached to its right side and color code dial for different keys

Technically, the note tokens are color-coded "blown" carfuses fitted with inexpensive low-tolerance resistors as identifiers for 36 different notes plus five percussion sounds. When a marble (this system uses ping-pong balls) passes the infrared sensor in the top of a synthesizer unit, the synthesizer chip evaluates the network of up to four attached note tokens (resistors) to identify the encoded notes und plays the respective ("piano") sounds. Synthesizer units can be placed freely along the helix allowing some leeway regarding the timing of individual notes or chords.

\section{ARCHITECTURE COMPARISON}

Apart from the above-mentioned introduction of polyphony and the support of chords and percussion sounds, there are other differences between the two designs. With an outer helix diameter of about $45 \mathrm{~cm}$ the new system is significantly smaller than the $85 \mathrm{~cm} \times 85 \mathrm{~cm}$ footprint of the initial design, which makes it easier to handle, especially for young children, but reduces playback time. The closed pipes of the first system allow playback only of whole configurations while the open track of the second design allows partial playback. The support structure of the first design allows testing of complete bars only while the second design allows testing of arbitrary subsets of compositions. The construction of a song using the initial design has to proceed from the bottom up, which means music must be composed backwards, adding an additional challenge to the interaction. This problem is solved with the frame structure of the second design. As marbles rolling down an inclined track accelerate, steps must be taken to linearize playback speed. The aerodynamic "piston effect" resulting from the closed pipes of the first design suffices for this purpose while the open track of the second design made it necessary to mount 16 acetate brushes into the frames to repeatedly slow down rolling ping pong balls. Of the differences between the two designs, the opposite system architectures appear to be the most critical, each with its own advantages, disadvantages and sources of error. The communication bus of the first system has shown a (subjectively estimated) $10 \%$ communication error rate. This might result from the lack of shielding of the bus line, unreliable pin connections between pipe segments or more likely, from precision tolerances in electronic components, as some individual pipe segments appear to be more error-prone than others. There is a potential risk of data collision on the communication bus of the first design when multiple marbles are rolled to play canons. With one centralized sound synthesizer, however, all sound events are in good relative tune. The decentralized architecture of the second design uses no communication bus and hence shows no related problems. Due to the lack of a convenient tuning facility on the synthesizer chips and other electronic components' imprecision however, the synthesizer modules of the second system have considerable problems with relative pitch. In both cases we have tackled architecturerelated issues by over-producing system components and hand-picking those showing reliable communication i.e. best relative pitch.

\section{USER TESTS}

We have conducted two user tests with the second design, each with two (female) music students. Objective of the tests was to establish whether the concept of music sequencing is indeed applicable and beneficial to children's music education and, more generally, whether the conceptual translation between spatial relationships of physical objects and temporal relationships between musical events can be performed. The students of the first test were 5 and 6 years old, the students of the second test were 8 and 9 years old. Both tests were conducted by a music teacher with a thorough understanding of the sequencer. The task of both sessions was to introduce the concept of chords to the students, assembling the song Happy Birthday according to a color-coded chart using the color code dial shown in figure 5 . The duration of each test was about 50 minutes with the first 30 minutes focusing on 
marble track interaction and the remaining 20 minutes focusing on reproducing Happy Birthday on an electronic piano using chords. Despite the sequencer and especially the note tokens being relatively rough objects and sometimes hard to (dis)connect all four subjects appeared attracted to the system and took considerable interest in interacting with it without losing their focus on the teacher's guidance.

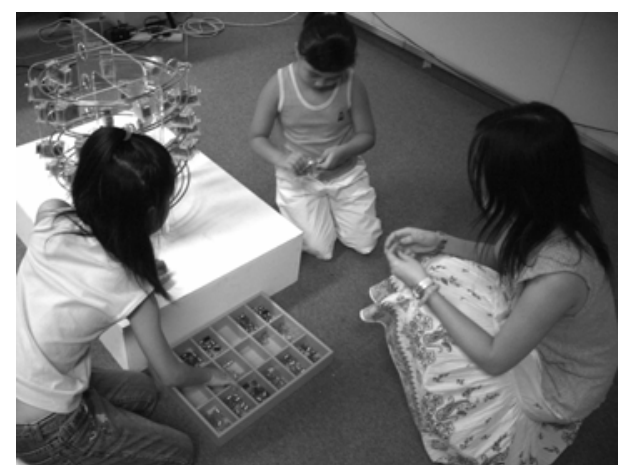

Fig. 6 User study involving two children and a music teacher conducting a lesson on chords

One student complained about the limited track length which is equivalent to about 12 seconds. As suggested by the usefulness of other color-coded materials for music education, the color-based notation of our system was useful and grasped by all students. Every translation between different representations of musical events (diagrams, scores, colored marble track tokens, piano keyboard) however appeared to require mental effort on top of the efforts necessary to understand melody making and sequencer interaction. The separation of the latter two has appeared useful in supporting music learning while minimizing frustrating learning experiences. All four students have shown comparable levels of understanding of the required interaction patterns and musical concepts and, with some variation in their confidence levels and playing speed, all were able to reproduce chord-based music on the piano at the end of the lesson. Rather unexpectedly, we observed older children repeatedly helping younger children using the marble track with respect to physical interaction as well as with respect to conceptual comprehension. The apparent ease with which even the younger students grasped the translation between physical configuration and temporal events suggest two hypotheses that should be tested in future work. Firstly, even younger users might be able to make this connection easily and benefit from this approach in their music education. Secondly, it might justify expecting users (including more mature ones) to perform similar conceptual translations between spatial and temporal arrangements also in other types of applications in the wider field of haptic programming.

\section{CONCLUSIONS}

Marbles rolling through three-dimensional structures allow the translation of physical configurations into sequenced temporal events in a way that is comprehensible to children and beneficial to their music learning experiences. Systems of this kind seem appropriate in the context of primary school and possibly also pre-school children's music education. Both the concept of marble tracks and that of construction kits relate directly to children's active play experience and are understood intuitively. Offering children physical music sequencers appears helpful in separating learning acts related to instrument manipulation and to melody making. This can be helpful where the simultaneous learning of both acts is experienced as frustrating. Marble track sequencers seem appropriate to support active acquisition of theoretical concepts of music. We have presented two alternative technical systems for this purpose, which are based on opposite systemarchitectural concepts, each with its specific advantages and disadvantages. With respect to mechanical execution, fully open tracks that allow (multiple) marble placement and pick-up at arbitrary points, appear highly appropriate to allow much richer experiences including simultaneous interaction by multiple users and the partial playback of compositions and canons but pose challenges regarding the linearization of playback speed.

\section{ACKNOWLEDGMENTS}

We acknowledge the support from our colleagues at our school, in particular E. Spicciolato, T. Jachna and J. Heskett. We are indebted to LAU Mei Ki who made the first marble track. We thank student helpers Chow Man Shan, Fu Wai Chan, So Lai Man and Wong Mei Ki for model making and Chow Wai Sze for model making, illustration and conducting user tests. This project is supported by HKPU (SD) CRG funds (G-YE40) and a CERG Grant from HK Research Grant Council (B-Q628).

\section{REFERENCES}

1. Eames, D. 901: After 45 Years of Working. The Voyager Company, Santa Monica, 1991.

2. Fischer, T. Teaching programming for and with Mircocontroller-Enhanced Physical Models. International Journal of Architectural Computing 1, 2, 58-74.

3. Fischer, T. and Frazer, J. H. Modeling architecture as logic states in space and time: History and future of the Universal Constructor. In: Chiu, M. L. (ed.): Insights of Smart Environments. CAAD Talks 5 (Archidata, Taipei 2005) 87-104.

4. Frazer, J. H. An Evolutionary Architecture. Architectural Association, London, 1995.

5. Frazer, J. H., Frazer, J. M. and Frazer, P. A. New developments in intelligent modelling. In Computer Graphics 81 (Online Publications, 1981) 139-154.

6. Personal and Ubiquitous Computing 8, 5 (entire issue).

7. Ullmer, B. and Ishii, H. Emerging frameworks for tangible user interfaces. IBM Systems Journal, 39, 3\&4 (2000) 915-931. 